\title{
2L-MAC: A MAC Protocol with Two-Layer Interference Mitigation in Wireless Body Area Networks for Medical Applications
}

\author{
Guan-Tsang Chen†, Wen-Tsuen Chen†t, and Shan-Hsiang Shen $\ddagger$ \\ $\dagger$ Department of Computer Science, National Tsing Hua University, Hsinchu 300, Taiwan \\ $\$$ Institute of Information Science, Academia Sinica, Nankang, Taipei 115, Taiwan \\ Emails: u9662320@oz.nthu.edu.tw, chenwt@iiis.sinica.edu.tw, sshen3@iis.sinica.edu.tw
}

\begin{abstract}
This paper investigates the issue of interference mitigation in wireless body area networks (BANs). Although several approaches have been proposed in BAN standard IEEE 802.15.6, they increase transmission latency or energy cost, and do not mitigate interference effectively. In order to avoid both intra- and inter-BAN interference, we present a MAC protocol with two-layer interference mitigation (2L-MAC) for BANs. Considering the QoS requirements of BANs, the proposed protocol not only avoids packet collisions but also reduces transmission delay and energy consumption in sensors. Moreover, channel switching is triggered whenever a BAN needs to acquire more bandwidth. Simulation results show that our protocol outperforms other protocols in terms of delivery rate, latency and energy saving.
\end{abstract}

Keywords-Body Area Network, BAN, MAC, Interference, Polling, IEEE 802.15.6

\section{INTRODUCTION}

With the developments in wireless communication and sensing techniques, wireless sensor networks (WSNs) have been widely adopted in various applications such as security, military, entertainments, and healthcare. According to different applications, sensors are deployed to gather different physical information. Some of them are put into human bodies to collect physiological and biological parameters such as body temperature, electrocardiogram (ECG), blood pressure, and respiratory rate, etc. The sensors deployed around a human body are referred to a body area network (BAN). The applications of BANs are quite diverse, including healthcare, elderly support, and sports assistance, etc. To standardize the BAN communication protocol, IEEE 802.15 Task Group has introduced a specification known as IEEE 802.15.6 [1].

A BAN is usually a star-like topology including a hub and a number of sensor nodes. The communication range of a BAN is limited to a few meters with data rate ranging from 100bps to $1 \mathrm{Mbps}$. Information is collected by the sensors equipped with radios and sent to the hub via wireless medium. By analysing these data from sensors, the hub can derive highlevel information (e.g., the health conditions of patients, movement types) and accordingly perform corresponding actions. The hub could also upload the received data to storage on clouds and acquires further services. To complete these tasks, the hub must possess adequate computation and communication capabilities. Usually a hub is a mobile device such as a smart phone or a PDA.

Due to human mobility, interference in networks is a critical issue in BANs [2] [3]. Equipped on human body, a BAN can move with the user. A number of BANs may stay close to each other, thus causing inter-BAN interference. Since there is no central coordinator among the co-located BANs, it is possible that two sensor nodes of different BANs transmit concurrently. When operating in the same channel, the BANs may suffer from packet collisions (as illustrated in Fig.1).

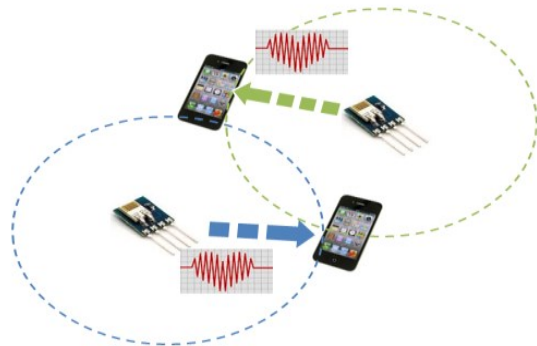

Fig. 1. The co-located BANs may suffer from inter-BAN interference if they operate in the same channel.

In IEEE 802.15.6 standard, there are three approaches suggested to avoiding inter-BAN interference, namely Channel Hopping, Beacon Shifting and Active Superframe Interleaving [1]. In channel hopping, a BAN periodically changes its operating channel according to a pre-defined hopping sequence. According to the IEEE standard, there are seven available frequency bands in narrowband ${ }^{1}$ and each of them contains 10 79 channels. By spreading traffic flows among the available channels, channel hopping may be effective in interference mitigation. However, due to the limited number of channels, if multiple BANs hop to the same channel, interferences still occur. Besides, since the hopping information is delivered to the sensors via beacons, if a beacon gets missed, the BAN may lose connectivity and need additional overhead to recover. Since a beacon usually carries important network information, beacon shifting is performed to prevent beacons from being corrupted frequently. In beacon

\footnotetext{
${ }^{1}$ In IEEE 802.15.6 standard, there are three frequency divisions including narrowband, ultra wideband and human body communication. Most medica applications operate in narrowband.
} 
shifting, a hub does not always transmit a beacon at the start of a beacon period. Instead, it divides a beacon period into four segments and sends beacons at the start of the different segments in the successive beacon periods. However, beacons from different BANs may still collide since each BAN does not know the shifting patterns of others. Active superframe interleaving enables the co-located hubs to communicate with each other to allocate channel bandwidth. After negotiation among the hubs, each BAN acquires a collision-free duration for transmission. Unfortunately, neither specific rules for bandwidth allocation nor the inter-BAN communication protocol is defined in current IEEE 802.15.6 standard.

The protocol proposed in [5] transforms a BAN interference problem into a graph coloring problem, where the nodes in a graph stand for BANs and the colors represent resources such as channels or timeslots. If two BANs are located in the radio coverage of each other, the corresponding two nodes in the graph are linked by an edge to indicate that they cannot be assigned the same color. The algorithm consists of two phases. In phase one, a hub communicates with its neighbor hubs to contend for resources. In phase two, each BAN performs transmission using the resources acquired in phase one. Although the protocol seems to alleviate the interference among BANs, it is impractical to be used in mobile BANs because the resource allocation may conflict whenever the BAN topology changes. In $\mathrm{B}^{2}$ IRS [6], a hub keeps its radio on to detect the existence of other BANs. Once it receives a beacon from a neighboring BAN, it reschedules its active period after the end of the neighboring BAN's active period. Thus, BANs operating in the same channel would not overlap their active periods. However, if there are too many BANs staying closely, such rescheduling of active period can lead to significant transmission delay. Therefore, the protocol is not applicable to applications with real-time requirement.

Quality of service (QoS) is a very important issue in BANs designed for medical applications [4] [7]. So far, few protocols can deal with the inter-BAN interference and also consider the QoS issue. Considerations of QoS in BANs should include transmission latency and available bandwidth [9] [11] [12]. Table I shows the QoS requirements in terms of bandwidth and latency of several medical applications. Due to limited channel capacity, when multiple BANs coexist in the same channel, a BAN may not be able to acquire enough bandwidth to meet the QoS requirements of applications. To address the QoS issue, a MAC-layer protocol could assign priorities to the sensors according to their tolerance of transmission latency [14]. If the bandwidth is not sufficient, a sensor with higher priority must be the first one allowed to transmit.

In this paper, we propose 2L-MAC to deal with the interference problem of BANs in two layers. In the first layer, a hub employs polling to coordinate its intra-BAN transmissions. Thus, sensor nodes in a BAN do not transmit simultaneously. In the second layer, before polling a sensor node, a hub performs carrier sensing to avoid packet collisions among coexisting BANs. By adopting this two-layer solution, the proposed protocol simultaneously mitigates intra- and
inter-BAN interference. In addition to interference mitigation, we also consider the QoS requirements of various medical applications. Moreover, the protocol can be compatible with IEEE 802.15.6 standard.

Table I. QOS REQUIREMENTS OF DIFFERENT MEDICAL APPLICATIONS

\begin{tabular}{|c|c|c|}
\hline Application & Data rate & Latency \\
\hline ECG (3 leads) & $2.4 \mathrm{Kbps}$ & $<300 \mathrm{~ms}$ \\
\hline ECG (5 leads) & $10 \mathrm{Kbps}$ & $<300 \mathrm{~ms}$ \\
\hline Heart rate & $2 \mathrm{Kbps}$ & $<300 \mathrm{~ms}$ \\
\hline Blood pressure & $120 \mathrm{bps}$ & $<3 \mathrm{~s}$ \\
\hline Temperature & $120 \mathrm{bps}$ & $<3 \mathrm{~s}$ \\
\hline $\mathrm{SpO}_{2}$ & $120 \mathrm{bps}$ & $<3 \mathrm{~s}$ \\
\hline
\end{tabular}

The rest of the paper is organized as follows. We present the proposed protocol in Section II and the simulation results in Section III. Conclusions are given in Section IV.

\section{PROPOSED PROTOCOL}

There are three MAC protocols specified in IEEE 802.15.6 standard, namely CSMA/CA, Slotted Aloha, and TDMAbased polling. The contention-based protocols, CSMA/CA and Slotted Aloha, usually incur more traffic collisions, overhearing, and transmission delay. Besides, they may suffer from congestion due to the traffic correlation in BANs [4] [8]. Among these three protocols, the TDMA-based polling is a better choice to be used in BANs to meet various QoS requirements. However, the polling protocol defined in the IEEE standard is not able to avoid inter-BAN interference. In our proposed protocol, we adopt polling to coordinate intraBAN traffic and employ a carrier sensing mechanism to deal with inter-BAN interference. Before polling a sensor node, a hub must perform carrier sensing to check the channel state. If the channel is idle, the hub transmits a polling frame to the sensor nodes. Upon receiving the polling frame, a sensor node transmits its data to the hub. Since a hub always checks the channel state before performing polling, it does not affect the ongoing transmissions of neighboring BANs. Thus, packet collisions are significantly reduced. If the channel is busy, the hub holds the polling and performs backoff. To prevent multiple hubs from concurrently transmitting their polling frames when a busy channel becomes idle, we further use contention window $(C W)$ and backoff timer $(B T)$ to implement the backoff mechanism of BANs. Furthermore, to guarantee QoS requirements of sensors, our backoff mechanism considers both the sensor priority of an application type and current transmission delay caused by backoff, which are denoted as $P_{a p p}$ and $T_{\text {delay }}$ respectively.

Before describing the proposed protocol, we first define the priority mechanism to implement QoS for the BANs. In IEEE 802.15.6 standard, there is a priority mapping according to the application type as shown in Table II, and we use this mapping to determine the value of $P_{a p p}$. As shown in Table III, there is a contention window for a corresponding priority of a sensor node. The application with higher priority is assigned a smaller $C W$, which leads to a shorter backoff duration, and the hub thus has a better opportunity to poll the sensor. 
Table II. THE APPLICATION PRIORITY DEFINED IN IEEE 802 15.6 STANDARD

\begin{tabular}{|c|c|l|}
\hline Priority & $\boldsymbol{P}_{a p p}$ & \multicolumn{1}{c|}{ Traffic designation } \\
\hline Lowest & 0 & Background \\
\cline { 2 - 3 } & 1 & Best effort \\
\cline { 2 - 3 } & 2 & Excellent effort \\
\cline { 2 - 3 } & 3 & Video \\
\cline { 2 - 3 } & 4 & Voice \\
\cline { 2 - 3 } & 5 & Medical data or network control \\
\cline { 2 - 3 } & 6 & High-priority medical \\
\cline { 2 - 3 } & 7 & Emergency or medical implant event report \\
\hline
\end{tabular}

Table III. RANGE OF CONTENTION WINDOW WITH DIFFERENT PRIORITY

\begin{tabular}{|c|c|c|c|}
\hline $\boldsymbol{P}_{a p p}$ & $\boldsymbol{C W}$ & $\boldsymbol{P}_{a p p}$ & $\boldsymbol{C} \boldsymbol{W}$ \\
\hline 0 & {$[16,64]$} & 4 & {$[4,16]$} \\
\hline 1 & {$[16,32]$} & 5 & {$[4,8]$} \\
\hline 2 & {$[8,32]$} & 6 & {$[2,8]$} \\
\hline 3 & {$[8,16]$} & 7 & {$[1,2]$} \\
\hline
\end{tabular}

The proposed protocol also enables BANs to switch their operating channel. Once the backoff threshold $H_{\text {switch }}$ expires, both the hub and the sensors switch to another channel to acquire more channel bandwidth. The protocol is described in detail in the following subsections and presented in Algorithm 1 and Algorithm 2.

\section{A. Polling with Backoff Mechanism}

We assume that the hub has the knowledge of transmission period and the size of corresponding data from each polled sensor. Based on the sensor requirements, the hub is able to generate a polling schedule for triggering the polling events. To keep synchronization between the hub and the sensor nodes, a polling frame also carries timing information to notify the sensor nodes of the next polling time. When receiving a polling frame, a sensor node can determine when to wake up for the next polling. The proposed protocol based on polling with backoff mechanism is described as the following steps:

Step1 Once a polling event to node $n$ is triggered, the hub first performs $\mathrm{CCA}^{2}$ to check the channel state. If the channel is idle, the hub sends a polling frame to node $n$ and goes to step 3. Otherwise, the hub performs backoff as described in step 2.

Step2 In the beginning of the backoff procedure, the hub first determines the priority $P_{a p p}$ of node $n$ and sets the $C W$ size for the node according to Table III. It then randomly chooses an integer from $C W$ as the value of $B T$ and waits for the channel to become idle. After the channel is sensed idle for SIFS (short interframe space) period, the hub decreases $B T$ by 1 in each timeslot and suspends $B T$ when the channel becomes busy. When $B T$ reaches zero, the hub sends a polling frame to node $n$ and goes to step 3 .

Step3 After transmitting a polling frame, the hub waits to receive data frames from node $n$. If the hub does not receive the data frames within SIFS period, it assumes

${ }^{2} \mathrm{CCA}$ : clear channel assessment, a technique used to estimate channel state in PHY layer. that node $n$ does not receive the polling frame and goes back to step 1 to perform polling again.

When the hub receives the data frames from node $n$, some of the frames may be corrupted due to channel fading or other interference. In such cases, the hub needs to poll for the corrupted frames. If the hub receives all the data frames from node $n$, it then sends an ack frame to the node. Upon receiving the ack frame, node $n$ turns off the radio and sleeps until the next polling period. As an example of the proposed protocol, refer to Fig. 2. At the time of the first polling, since the channel is sensed idle, the hub does not perform backoff. However, before the second polling, the hub performs backoff for busy channel. We assume that $B T$ is initially set to 6 . After the channel is sensed idle for SIFS period, the hub starts to decrease $B T$. At the time when $B T$ counts down to 2 , the channel again becomes busy and $B T$ is suspended. After the channel becomes idle for SIFS period, the hub restarts to decrease $B T$. When $B T$ reaches 0 , the hub performs polling.

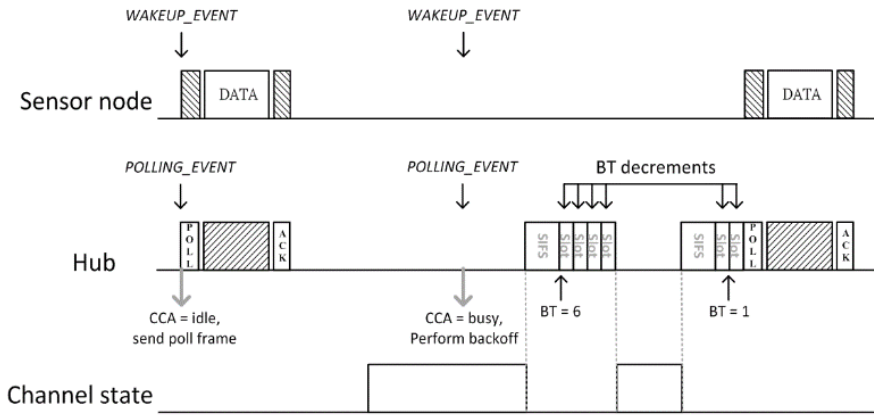

Fig. 2. An example of the proposed backoff mechanism.

In order to avoid that the backoff time exceeds the application deadline, we use a timer $T_{\text {delay }}$ to monitor the delay caused by the backoff. When a polling event is triggered, the hub starts the timer $T_{\text {delay }}$. Thereafter, the hub resets $T_{\text {delay }}$ only when it receives all the data frames from the polled sensor node. If $T_{\text {delay }}$ exceeds the predefined threshold $H_{\text {backoff }}$, the hub stops backoff even if $B T$ does not reach zero. Instead, the hub is allowed to transmit the polling frame whenever the channel is sensed idle for SIFS period.

\section{B. Channel Switching}

To perform channel switching, except for the hub, each sensor node needs to maintain a timer $T_{\text {delay }}$. When a wake-up event is triggered, the sensor starts $T_{\text {delay }}$ until it receives a polling frame from the hub. If $T_{\text {delay }}$ exceeds the predefined threshold $H_{\text {switch }}$, both the hub and the sensor node switch from the current operating channel to a backup channel. A backup channel is chosen by the hub and specified in the previous polling frame. After performing channel switching, the hub chooses the next channel from the pre-generated channel sequence as a new backup channel. The updated channel information is delivered to the sensor via the next polling frame. From the received polling frames, the sensor node can always be aware of the operating channel and the backup channel. A hub confirms that a sensor does receive the 
updated channel information by receiving an ack frame from the sensor node. A channel sequence can be generated using a 16-bit Galois linear feedback shift register (LFSR) function which is defined in IEEE 802.15.6 standard.
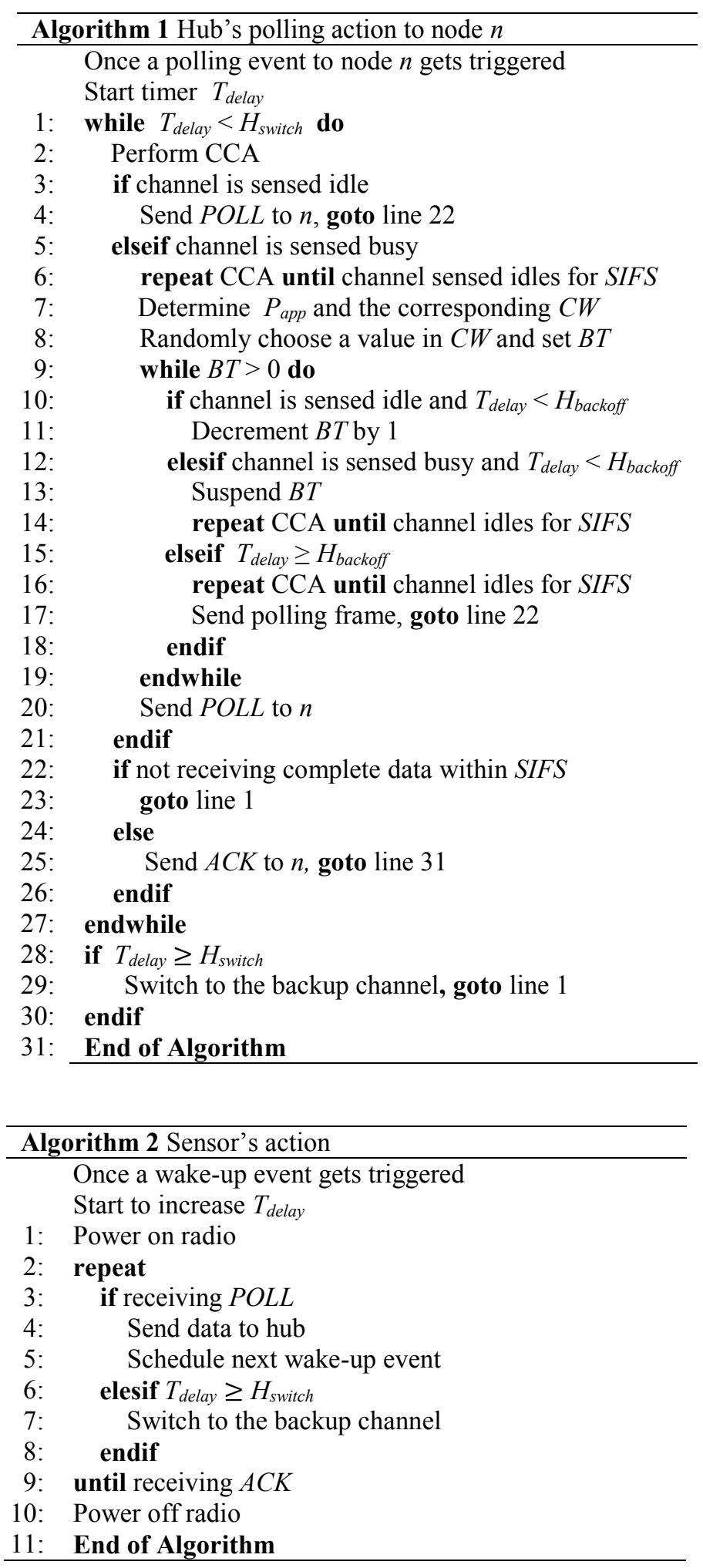

\section{SIMULATION RESULTS}

The simulation results are obtained by using $\mathrm{OMNeT}++$ simulator [10]. We use MixiM network model [13] based on $\mathrm{OMNeT}++$ simulator to implement our $\mathrm{BAN}$ radio transceiver. Parameters in the physical layer are set according to narrowband PHY specification in IEEE 802.15.6 standard. The remaining parameters are listed in Table IV. To evaluate the proposed protocol under dense BANs with long-term interference, we place BANs close together and make them static so that each BAN is always located in the interference range of others. Transmission period of each BAN is set to $0.2 \mathrm{~s}$. Since the transmission modulation in physical layer varies with the channel quality. According to the IEEE standard, we choose $250 \mathrm{Kbps}$ as the average PHY data rate. Our performance metrics in the following simulations include delivery rate, latency, and energy consumption in sensors.

Table IV. SIMULATION PARAMETERS

\begin{tabular}{|c|c|}
\hline Parameter & Value \\
\hline Data rate & $250 \mathrm{Kbps}$ \\
\hline Tx power & $-15 \mathrm{dBm}$ \\
\hline Tx energy cost & $2.9 \mathrm{~mW}$ \\
\hline Rx energy cost & $3.1 \mathrm{~mW}$ \\
\hline Radio sensitivity & $-87 \mathrm{dBm}$ \\
\hline Transmission period & $0.2 \mathrm{~s}$ \\
\hline
\end{tabular}

First, we show the performance of the proposed protocol in interference mitigation when BANs operate in the same channel. Here we do not enable the channel switching mechanism and BANs operate in a single channel. We first compare the proposed protocol with the original polling protocol specified in the IEEE standard. Fig. 3 depicts the delivery rate vs. data size for various number of co-channel BANs. By employing carrier sensing and a backoff mechanism, our protocol achieves around $80 \%$ delivery rate when the data size is less than 6 Kbits. In contrast, since the original polling protocol does not check the channel state before polling, it suffers severe data loss as the number of BANs and data size increase. Fig. 4 shows the delivery latency in two protocols. Since transmissions occur every $0.2 \mathrm{~s}$, for those data frames that cannot reach the destination within $0.2 \mathrm{~s}$, we treat them as timeout frames and set their delivery latency to $0.2 \mathrm{~s}$. As the figure depicts, $2 \mathrm{~L}-\mathrm{MAC}$ has shorter delivery latency in comparison to the original polling protocol. In most cases, 2L-MAC guarantees the latency of less than $125 \mathrm{~ms}$.

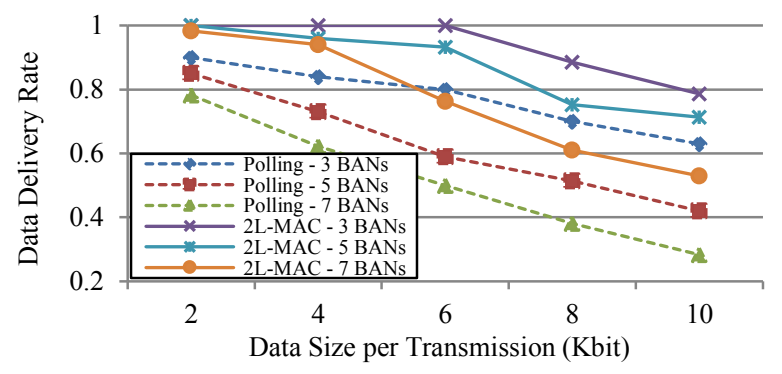

Fig. 3. Comparison of delivery rate between the proposed protocol and the original polling protocol 


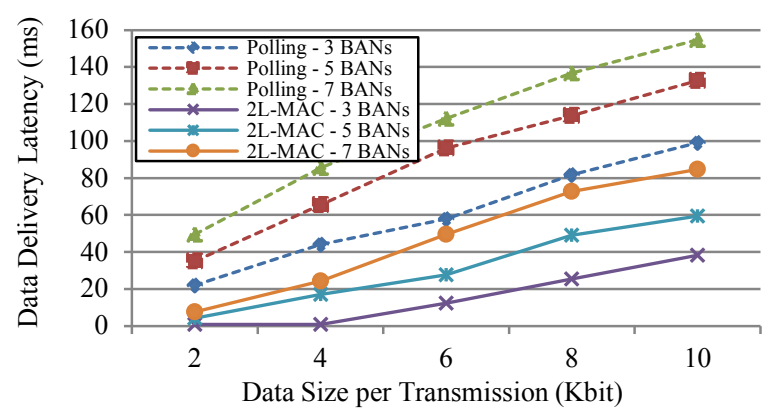

Fig. 4. Comparison of delivery latency between the proposed protocol and the original polling protocol

We also compare the proposed protocol with $\mathrm{B}^{2} \mathrm{IRS}[6]$ and the beacon shifting scheme presented in the IEEE standard [1]. By employing TDMA-based polling, there is no intra-BAN interference in 2L-MAC. The other two protocols do not mention how they coordinate the intra-BAN traffic. To be fair, we assume that intra-BAN interferences in the protocols do not occur. The comparison is made under dense BANs, where the number of co-channel BANs is set to 7. The comparisons of delivery rate, latency and energy consumption for the three protocols are shown in Fig. 5, Fig. 6 and Fig. 7 respectively. In $B^{2}$ IRS, a hub reschedules its network active period whenever it receives beacons from neighboring BANs. Although such rescheduling of active period can avoid packet collisions, it incurs more transmission delay. Whenever a hub performs rescheduling, it causes delay to all the following transmissions. Thus, a number of timeout frames cause a poor delivery rate. In the beacon shifting scheme, since each BAN does not know the shifting pattern of other BANs, packet collisions occur randomly. If a beacon is lost, the sensors cannot be aware of the timeslot allocation in the following beacon period, thus causing the transmission failures. As illustrated in Fig. 5 and Fig. 6, the proposed protocol outperforms the other two protocols in both delivery rate and latency. Fig. 7 shows the comparison of sensor's energy consumption per time unit. In our simulation, a sensor node turns off its radio only when it receives an ack frame from the hub. Due to transmission delay, sensor nodes may consume additional energy on their radios. From Fig. 7, 2L-MAC reduces the sensor's energy consumption by $24.2 \%$ and $42.7 \%$ respectively in comparison to beacon shifting and $\mathrm{B}^{2} \mathrm{IRS}$. In terms of energy consumption per bit, since 2L-MAC achieves higher delivery rate, 2L-MAC reduces the sensor's energy consumption by $44.1 \%$ and $78 \%$ respectively compared to beacon shifting and $\mathrm{B}^{2} \mathrm{IRS}$.

When there are multiple channels available, the proposed protocol allows BANs to change their operating channel by channel switching. By comparing the performances of $2 \mathrm{~L}$ MAC with/ without a channel switching mechanism, we can see the improvement made by channel switching. According to the IEEE standard, each frequency band in the narrowband has at least 10 channels. Thus, we set the number of available channels to 10. The simulation results are shown in Fig. 8 and Fig. 9. In the simulations, we assume that all the BANs operate initially in the same channel. With the channel switching mechanism, when the transmission delay exceeds the threshold $H_{\text {switch }}$, a BAN switches its operating channel. Due to the decrease of co-channel BANs, the delivery performance of the scheme with the channel switching mechanism does not degrade sharply when the data size increases.

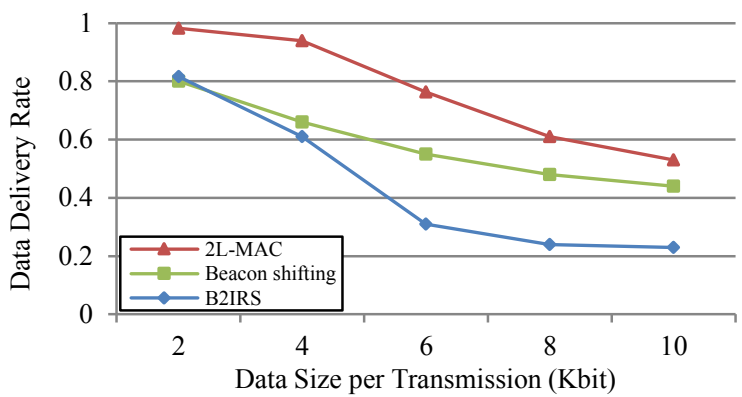

Fig. 5. Average delivery rate of the sensor nodes in three protocols.

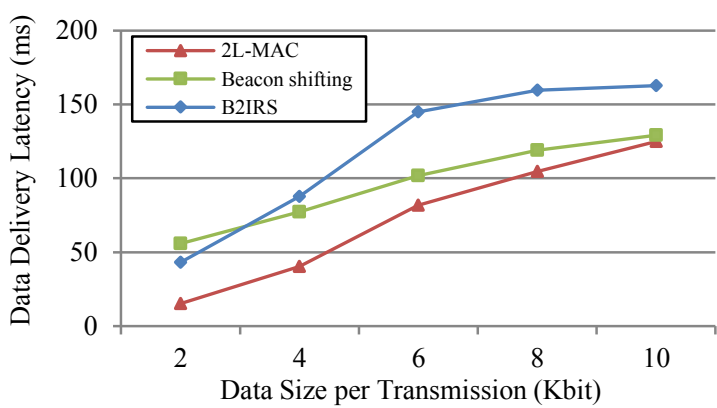

Fig. 6. Average delivery latency of the sensor nodes in three protocols.

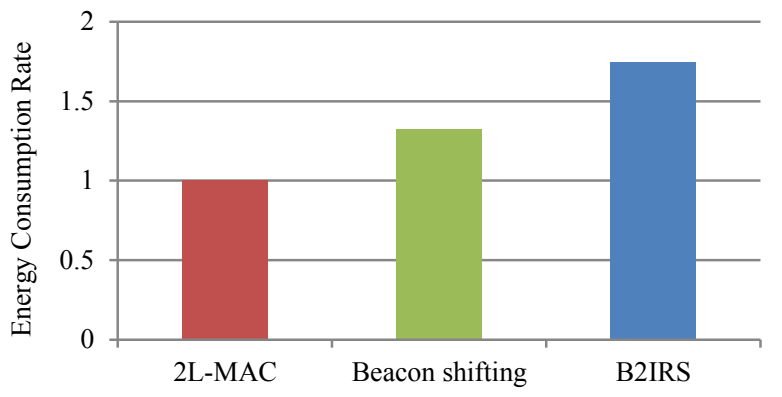

Fig. 7. Energy consumption rate of the sensor nodes in three protocols.

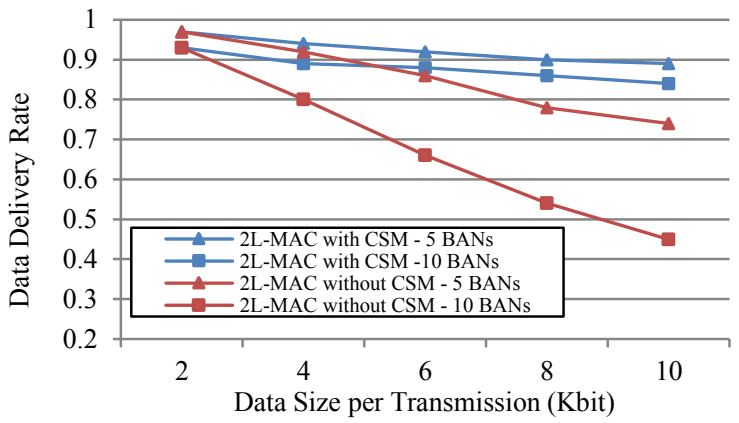

Fig. 8. Data delivery rate with channel switching mechanism enabled. 


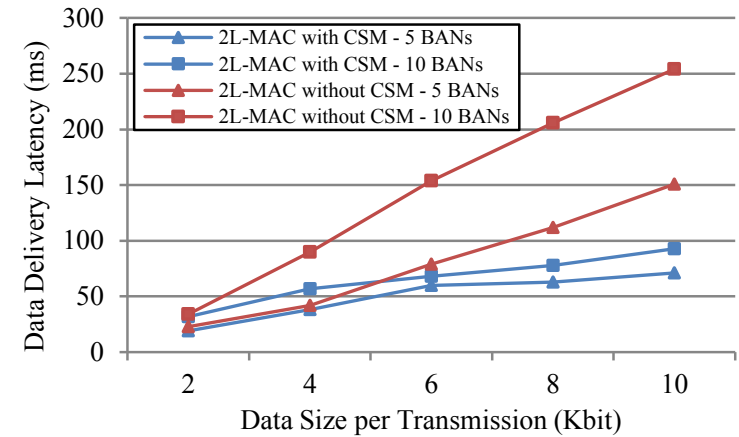

Fig. 9. Data delivery latency with channel switching mechanism enabled.

We then compare the proposed protocol with the channel hopping scheme presented in the IEEE standard. Likewise, we assume that there are 10 available channels in the frequency band. The number of co-located BANs is set to 10, 20 and 30 for various densities of BANs. We assume that the BANs are randomly distributed among the channels. As illustrated in Fig. 10 , the proposed protocol reduces as much as $23 \%$ of packet collisions in comparison to the channel hopping scheme. When there are 30 BANs, the proposed protocol still has delivery rate more than $85 \%$ better. Although the channel hopping scheme could reduce packet collisions by periodically changing the operating channel, however, without carrier sensing, packet collisions occur when BANs hop to the same channel.

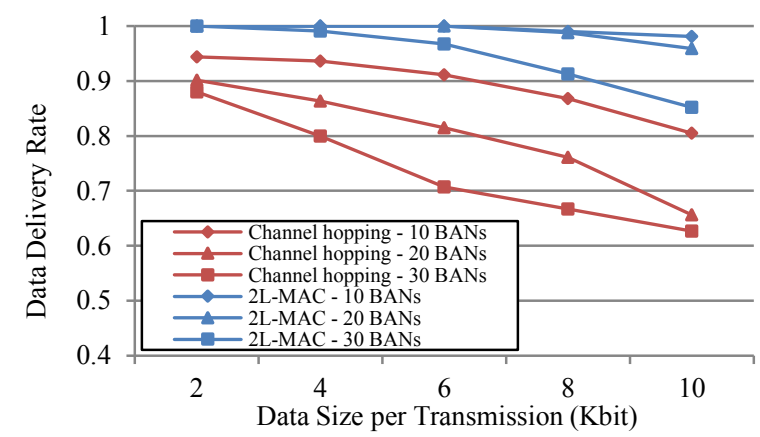

Fig. 10. Comparison of our protocol and the channel hopping defined in the IEEE standard.

According to our simulation results, when 20 BANs operate in 10 channels, the proposed protocol ensures that each BAN acquires the bandwidth of $50 \mathrm{Kbps}$ (10Kbit every $0.2 \mathrm{~s}$ ) with $95 \%$ delivery rate and latency less than $80 \mathrm{~ms}$. Considering the requirements of medical applications listed in Table I, being allocated with bandwidth of $50 \mathrm{Kbps}$, each BAN in the proposed protocol may contain more than 4 sensors. In other words, given 10 available channels, the proposed protocol can support more than 80 sensors (20 BANs with 4 sensors each) and satisfy their QoS requirements at the same time. As for the performance of interference mitigation, IEEE 802.15.6 standard requires a BAN protocol supporting at least 60 sensors in a $6^{3} \mathrm{~m}^{3}$ space. In the narrowband, each frequency band consists 10 79 channels. With only 10 channels available, 2L-MAC still meets the requirement specified in the IEEE standard.

\section{CONCLUSIONS}

In this paper, we present $2 \mathrm{~L}-\mathrm{MAC}$, a distributed pollingbased protocol where a hub makes carrier sensing before sending a polling frame and performs backoff once the channel is sensed busy to avoid inter-BAN interference. We implement sensor priorities for satisfying QoS requirements and make BANs change their operating channels to acquire more bandwidth by performing channel switching. In the simulations, we compare 2L-MAC with other approaches designed to avoid inter-BAN interference including beacon shifting, channel hopping and $\mathrm{B}^{2}$ IRS. The results show that the proposed protocol outperforms these approaches both in delivery performance and energy efficiency. Therefore, $2 \mathrm{~L}$ MAC can be applicable to medical applications. Our future works include consideration of unstable channel quality in moving BANs. Moreover, 2L-MAC will be implemented on sensor platforms based on IEEE 802.15.6 and applied to real medical application environments.

\section{REFERENCES}

[1] IEEE 802.15 WPAN Task Group 6. Available online: http://www.ieee802.org/15/pub/TG6.html

[2] H. Viswannthan, B. Chen and D. Pompili, "Research Challenge in Computation, Communication, and Context Awareness for Ubiquitous Healthcare," IEEE Communication Magazine, pp. 92-99, May 2012.

[3] A Bolis, D. Smith, D. Miniutti, L. Libman, and Y. Tselishchev, "Challenges in body area networks for healthcare: the MAC," IEEE Communications Magazine, vol. 50, no. 5, pp.100-106, 2012.

[4] H. Li and J. Tan, "Heartbeat Driven Medium Access Control for Body Sensor Networks," Proceedings of the 1st ACM SIGMOBILE international workshop on Systems and networking support for healthcare and assisted living environments, pp. 25-30, Jun. 2007.

[5] S. Cheng and C. Huang, "Coloring-Based InterWBAN Scheduling for Mobile Wireless Body Area Network," IEEE Transactions on Paralle and Distributed Systems, vol.14, pp. 250-259, Apr. 2012.

[6] P. R. Grassi, V. Rana, I. Beretta and D. Sciuto, "B²IRS: A Technique to Reduce BAN-BAN Interferences in Wireless Sensor Networks," Proceedings of the International Conference on Wearable and Implantable Body Sensor Networks, pp. 46-51, May 2012.

[7] S. Cheng, C. Huang and C. Tu, "RACOON: A Multiuser QoS Design for Mobile Wireless Body Area Networks," Journal of Medical Systems, vol. 35, pp. 1277-1287, 2011.

[8] H. Li and J. Tan, "An Ultra-low-power Medium Access Control Protocol for Body Sensor Network," Proceedings of the 2005 IEEE Engineering in Medicine and Biology 27th Annual Conference, Shanghai China, Sep. 2005

[9] C. Tachtatzis, F. Franco, D. Tracey, N. Timmons and Jim Morrison, “An Energy Analysis of IEEE 802.15.6 Scheduled Access Modes for Medical Applications," Proceedings of the Third International ICST Conference, ADHOCNETS 2011, Paris, France, Sep. 2011

[10] A. Varga, "The OMNeT++ Discrete Event Simulation System," Proceedings of the European Simulation Multi-Conference, Czech Republic, Jun. 2001

[11] D. B. Smith, D. Miniutti, and L.W. Hanlen, "Characterization of the Body-Area Propagation Channel for Monitoring a Subject Sleeping," IEEE Transactions on Antennas and Propagation, pp. 4388-4392, 2011

[12] D. Kim and J. Cho, "WBAN meets WBAN: smart mobile space over wireless body area networks," Proceedings of the IEEE Vehicular Technology Conference, Fall, Anchorage, pp. 1-5, 2009.

[13] "MiXiM Network Simulation Model." Available online: http://mixim.sourceforge.net/

[14] B. Kim and J. Cho, "A Novel Priority-based Channel Access Algorithm for Contention-based MAC Protocol in WBANs," Proceedings of the 6th International Conference on Ubiquitous Information Management and Communication, Feb. 2012. 